\title{
AIAA 2004-0377 Applications of Computational Methods for Dynamic Stability and Control Derivatives
}

Lawrence L. Green

NASA Langley Research Center

Hampton, VA

Angela M. Spence

Mississippi State University

Mississippi State, MS

42nd AIAA Applied Aerospace Sciences

Conference and Exhibit

January 5-8, 2004

Reno, NV 


\title{
APPLICATIONS OF COMPUTATIONAL METHODS FOR DYNAMIC STABILITY AND CONTROL DERIVATIVES
}

\author{
Lawrence L. Green ${ }^{*}$ \\ NASA Langley Research Center, Hampton, VA \\ Angela M. Spence** \\ Mississippi State University, Mississippi State, MS
}

\begin{abstract}
Initial steps in the application of a low-order panel methed computational fluid dynamics (CFD) code to the calculation of aircraft dynamic stability and control $(S \& C)$ derivatives are documented. Several capabilities, unique to CFD but not unique to this particular demonstration, are identified and demonstrated in this paper. These unique capabilities complement conventional S\&C techniques and they include the ability to: 1$)$ perform maneuvers without the flow/kinematic restrictions and support interference commonly associated with experimental S\&C facilities, 2) easily simulate advanced $S \& C$ testing techniques, 3) compute exact $S \& C$ derivatives with uncertainty propagation bounds, and 4) alter the flow physics associated with a particular testing technique from those observed in a wind or water tunnel test in order to isolate effects. Also presented are discussions about some computational issues associated with the simulation of $S \& C$ tests and selected results from numerous surface grid resolution studies performed during the course of the study.
\end{abstract}

\section{INTRODUCTION}

Aircraft stability and control (S\&C) derivatives quantify the aerodynamic forces and moments used to model aircraft dynamics. S\&C derivatives are used to predict, for example, the longitudinal short period, lateral pure roll, lateral Dutch roll, spin behaviors, and handling qualities sensed by pilots for a given configuration. Historically, aircraft static and dynamic S\&C derivatives have been determined through wind tunnel tests, water tunnel tests, or with empirical formulations ${ }^{1-8}$ based on prior tests. However, most of the wind tunnels and all of the water tunnels used for $\mathbf{S} \& \mathrm{C}$ tests operate at low free stream velocities and Reynolds numbers. These limitations restrict the range of flight conditions that can be adequately simulated.

Many S\&C testing facilities provide unique dynamic capabilities that are not typically available in the wind tunnels used for aircraft performance tests.

This material is declared a work of the U.S. Government and is not subject to copyright protection in the United States.
Because of their dynamic capabilities, these S\&C facilities may be expensive to build, operate, and/or maintain. The range of motions that can be performed in such facilities may be limited by the wind tunnel walls or by the test rig's kinematic and vibrational restrictions. In addition, many conventional dynamic test facilities do not provide the capability to determine damping and cross derivatives individually; instead, these facilities can only provide measurements of combined derivatives. 9 The measurement of these quantities in pairs is not the preferred situation; it is simply a kinematic reality of the facilities available. A companion paper $^{10}$ details several computational techniques to separate such paired dynamic derivatives. Some facilities are not equipped with a "slip ring" capability and thus, cannot perform continuous rotary motions; instead they rely on oscillatory motions to provide brief periods of steady rotational motion.

The difficulty of many experimental S\&C facilities to adequately model the flight characteristics of today's aircraft over the entire flight envelope, the cost of developing better $\mathrm{S} \& \mathrm{C}$ testing facilities, and the recent advent of novel morphing aircraft configurations ${ }^{11-13}$ all contribute to the difficulty of predicting $S \& C$ derivatives. Prior research efforts ${ }^{12-29}$ using CFD to predict $S \& C$ derivatives have made some progress toward bridging the capability gap, but these efforts are very time consuming ${ }^{15}$ and have failed to make a significant impact in the day to day business of S\&C prediction. Because both CFD and experimental studies ${ }^{30.31}$ have their unique disadvantages, aerospace companies spend millions of dollars fixing S\&C problems discovered during certification flight tests or production use. The current situation for $\mathrm{S} \& \mathrm{C}$ prediction is similar to the situation seen before CFD methods were widely used to accurately predict aerodynamic performance.

CFD offers capabilities and techniques that complement experimental $S \& C$ testing techniques for obtaining S\&C derivatives. With CFD, support and

\footnotetext{
* Senior Research Scientist, Multidisciplinary Optimization Branch, M/S 159, Senior Member AIAA ${ }^{* *}$ Engineering Co-op Student, Multidisciplinary Optimization Branch, M/S 159, Student Member AIAA
} 
wall interference effects can be either modeled or eliminated in order to assess their impact. Because the vehicle orientations and movements within the flow can be arbitrarily specified to obtain time-dependent solutions, CFD can easily simulate advanced $\mathrm{S} \& \mathrm{C}$ testing techniques (possibly including those for which no testing facility currently exists). By applying various differentiation techniques ${ }^{12-16,32,33}$ to CFD codes, researchers can obtain exact derivatives of the forces and moments throughout the time history of a maneuver. CFD can independently eliminate or refine various physics features within a flow to isolate particular effects. Finally, CFD can help explain the causes and types of separated flows affecting S\&C prediction.

In theory, applying CFD to $\mathrm{S} \& \mathrm{C}$ prediction is not only feasible, but straight forwardl; one would simply grid the vehicle of interest and perform the required calculations. In practice, many issues must be addressed before CFD can accurately predict S\&C derivatives, including:

- the cultural differences that exist between the CFD and $S \& C$ communities, evidenced by differences in language, notation, and expectations of the two groups

- the significant increase in computer resources (execution time, memory, and disk space) required to address the aircraft $\mathrm{S} \& \mathrm{C}$ derivative needs ${ }^{15.16}$ beyond the substantial resources already required for standard aircraft $\mathrm{CFD}$ performance calculations

- the need for uncertainty quantification of S\&C derivatives used in flight simulations and multidisciplinary design efforts and the further increase in computational resources ${ }^{34-38}$ associated with computing $\mathrm{S} \& \mathrm{C}$ predictions with uncertainty included

- the absence of a set of acceptable test cases that the CFD community must solve and the degree of accuracy required for CFD to become a credible alternative to wind and water tunnel testing

To address these concerns, a team was recently formed at the National Aeronautics and Space Administration (NASA) Langley Research Center (LaRC). The Computation Methods for Stability and Control (COMSAC) team includes key researchers in the CFD and $\mathrm{S} \& \mathrm{C}$ communities devoted to developing and applying CFD methods for S\&C needs. A recent workshop hosted by the COMSAC team devoted to this topic was well attended, and the topic gained broad support from the aerodynamic community. The COMSAC team is currently examining many research endeavors in this arena. ${ }^{12-29}$ COMSAC's mission is to: understand the causes and types of separated flows affecting $\mathrm{S} \& \mathrm{C}$ prediction; use this understanding to develop computational methods to predict the S\&C derivatives of interest; and create a long-term plan for synergistic use of experimental facilities, testing techniques, and CFD methods to close the gap between CFD and experimental S\&C activities. This paper is among the first to be published under this new effort.

Results presented in this paper are relevant to furthering $\mathrm{S} \& \mathrm{C}$ applications of $\mathrm{CFD}$. The results are computed by using a low-order panel method code on a simplified F-16XL fighter configuration. This work represents the first of many steps needed in the application of CFD to S\&C problems. The configuration was chosen because it has a wide variety of measured data available. The panel code was chosen, among other reasons, for its quick run time, ease of use, extensive motion capabilities and its ability to represent three-dimensional bodies within timedependent flows.

In some ways, the use of a low-order panel method CFD code to compute the forces and moments of a highly swept configuration like the F-16XL at high angles of attack is a worst-case scenario. The vortical and viscous effects, which dominate the F-16XL flowfield, at high angles of attack, are not modeled in the code. The authors of this paper certainly do not advocate the uninformed use of a low-order panel method code for the computation of viscous, vortical, bursting-vortical, or separated flows. However, the code was used anyway because of the great potential to preliminary design that could result from a successful application. The focus of this paper is to demonstrate several computational techniques that advance the ability of CFD to compute S\&C derivatives; this paper also raises a number of significant issues associated with S\&C computations. The authors do advocate using the appropriate level of physics fidelity with respect to the problem posed, which can only be assessed by examining all available levels of fidelity. Producing quantifiable and justifiable error bounds on the accuracy of results is a critical area that must be addressed when using CFD to predict $S \& C$ derivatives. This paper demonstrates a grid resolution study and uncertainty propagation techniques that can be used as a model for providing error bounds in the future. Error bounds help identify the limitations of the current method; eventually, researchers will be able to compare the error bounds from all levels of fidelity and determine the required fidelity for any $S \& C$ application.

\section{TECHNICAL APPROACH}

This study involves the application of the automatic differentiation of FORTRAN (ADIFOR) tool, version 2.0D, ${ }^{40,41}$ to the Panel Method Ames Research Center (PMARC) code, ${ }^{42}$ version 14.10. ADIFOR 2.0D was applied to PMARC by using a wide 
variety of input variables as potential independent variables of differentiation. The PMARC body and wind axis forces and moments were used as the dependent variables of differentiation. Since uncertainty estimates of all S\&C parameters (forces and moments and their derivatives) are normally requested by most control law designers, the computation of both first and second derivatives with respect to selected independent variables of differentiation are demonstrated; the latter are used to enable the propagation of known or assumed input variable uncertainties through the code to determine the uncertainty effects on the computed forces and moments. The relationship for uncertainty propagation ${ }^{36.38}$ as a function of normally distributed. random variables is

$$
\sigma_{j}=\sum_{i=l}^{m}\left(\frac{\partial F_{j}}{\partial x_{i}} \sigma_{i}\right), j=l, n
$$

$m=$ number of random inputs

$n=$ number of output functions

$\sigma_{i}=$ standard deviation of

\section{the random inputs}

In this case, the output function $F$ may be any of the computed body or wind axis forces and moments, or their first derivatives with respect to parameters such as the angle of attack. angle of sideslip, and the steady rotational rates. Original and ADIFOR-generated PMARC code versions were executed for an array of oscillatory and rotary aircraft motions to compare with data generated for a $10 \%$ scale F-16XL model tested ${ }^{9}$ in the NASA LaRC 14- by 22-Foot Subsonic Tunnel. Other data from wind tunnel (18\% scale model) ${ }^{43}$ and water tunnel $(2.5 \% \text { scale model })^{44,45}$ tests is also available, but was largely ignored for this study. The computational F-16XL geometry, as shown in Figure 1, was modified from that of the actual aircraft. The engine inlet was faired over with a smooth, bulbous surface and some detail near the aft end of the aircraft was simplified; this same geometry was used in the water tunnel tests. Limited comparisons with experimental data ${ }^{9,3}$ are presented in this paper. Other configurations, including a generic transport wingbody. a Boeing 747 wing-body-pylon-nacelle configuration, and an F/A-18 A/B fighter were also executed during the course of the present studies; the results of these studies may be the subject of a followon publication. Only F-16XL data is shown in this paper.
Computational Fluid Dynamics Code

The demonstrations presented here employ the PMARC code, ${ }^{42}$ a low-order panel method CFD code that was modified by applying AD to enable efficient computation of exact first and second force and moment derivatives with respect to a wide variety of code inputs. This panel method CFD code was chosen for its fast execution and moderate computational resource requirements, ease of use, ability to simulate a wide variety of steady and time-dependent rotary and oscillatory aircraft motions, and its amenability to processing by $\mathrm{AD}$ to compute derivatives that are exact to the formulation accuracy of the code solution. Second derivatives have been included to allow for input variable uncertainty propagation through the code with respect to the output forces and moments and their first derivatives. From the combination of the above mathematical techniques and the panel method choice, a demonstration of the computation of aircraft dynamic S\&C derivatives, with propagated uncertainty bounds, could be rapidly performed. Sample results are presented for the F-16XL fighter configuration modeled with a coarse surface grid, shown in threeview in Figure 1.

The PMARC, ${ }^{42}$ version 14.10 potential flow solver is a FORTRAN 77 code that can compute surface pressures, forces, and moments of arbitrary shapes. The code assumes inviscid, irrotational, and incompressible flow. Boundary layer and compressible corrections are available but are not implemented in this study. PMARC can also compute solutions of unsteady, time-varying flow conditions for a wide variety of aircraft motions. The original PMARC code has been modified to allow for the input of a free stream Mach number, angle of attack, and angle of sideslip in degrees, rather than simply the input of free stream velocity components. The code was also modified to allow ADIFOR processing by replacing the use of scratch $\mathrm{I} / \mathrm{O}$ files with common block access, and by inserting a few lines of code into the program to identify independent and dependent variables of differentiation.

The input file to the program uses a set of grid points describing the shape of the geometry as a set of panels. In this study, both the right and left halves of a modified F-16XL configuration are modeled in the PMARC input geometry file. The gray-scale in Figure 1 reflects different surface patches in the PMARC geometry input file, however these patches cannot be readily identified from the figure. The surface resolution, with 984 surface points and 566 surface panels, is moderately coarse by CFD standards, yet the vehicle is still clearly identifiable. Higher surface resolution grid files were available and used. Several such studies were performed and are discussed in the sections "Grid Resolution Studies" and "Results". 
PMARC allows the user to describe only half the aircraft and mirror the solution in the $x-z$ plane. However. this technique does not capture the eflects of a nonzero angle of sideslip, which are of interest in the $\mathrm{S} \& \mathrm{C}$ arena. The input file also specifies the flight condition, certain algorithmic parameters, and the userdefined position of the reference point about which all moments are summed. The forces and moments are also nondimensionalized with a user-specified reference area, mean aerodynamic chord length, and wingspan.

The PMARC code includes a wide variety of possible aircraft motions:

- $\quad$ straight translation with imposed angles of attack $(\alpha)$ and sideslip ( $\beta$ )

- pure rotary motions about the three axes described as constant rotational rates ( $p$, $q$, and $r$ )

- planar oscillatory motions in both translation and rotation about each of three axes, each described by an amplitude and frequency

- coning motions (illustrated subsequently) used within advanced experimental S\&C facilities

In contrast to the limitations of some experimental facilities, coning motions can be simulated within PMARC with either continuous (i.e., a "slip ring") or limited roll motion capability (oscillatory test motion).

In addition, the authors also use two other capabilities of the CFD code that are not generally available to $S \& C$ experimentalists. These capabilities are: the choice of either rigid or flexible wakes, and the ability to somewhat arbitrarily prescribe the time stepping characteristics of the solution. Although flexible wakes are preferred because they more accurately model the flow physics involved, the rigid wakes were more robust for some of the motions of interest and also lend themselves better to flow visualization. Wakes are currently attached to the wing trailing edges and the upper vertical tail trailing edge. The time stepping characteristics of a given solution can be defined to match the data sampling rate of a particular S\&C facility, if desired. A caveat of this technique is that the size of the shed wake panels in the PMARC solution is proportional to the chosen time step, which alters the flow physics to some extent if sufficiently small time steps are not used.

\section{Automatic Differentiation}

\section{Automatic differentiation ${ }^{40,41,46}(\mathrm{AD})$ is a}

technique for derivative computation. $\mathrm{AD}$ relies on the fact that every function, no matter how complicated, is executed on a computer as a (potentially very long) sequence of elementary arithmetic operations and functions evaluations (i.e., sine or cosine). By repeatedly applying the chain rule of differential calculus to the composition of those elementary operations, derivative information can be computed exactly and automatically.

The two approaches for computing derivatives with $\mathrm{AD}$ are the forward mode and the reverse mode. The forward mode applies the chain rule of differentiation to propagate, equation by equation, derivatives of intermediate variables with respect to the input variables. In contrast, the reverse (adjoint) mode propagates, in reverse through the program, the derivatives of the output variables with respect to the input variables. The forward mode is better suited to problems with fewer input variables than output variables, whereas the reverse mode is better suited to problems with fewer output variables than input variables. Many hybrids of the forward and reverse modes are possible, with complementary tradeoffs in required random access memory (RAM), disk space, and execution time.

The forward method of $A D$ is implemented in the ADIFOR, version $2.0 \mathrm{D}^{40.41}$ tool. The reverse mode and some second derivative capability are also implemented in the ADIFOR, version 3.0. ${ }^{46}$ Both tools were developed jointly by the Center for Research on Parallel Computation at Rice University and the Mathematics and Computer Sciences Division at Argonne National Laboratory. Both tools have been used with the PMARC code, but only results using the ADIFOR 2.0D tool are shown in this paper. In general, to apply ADIFOR to a given FORTRAN 77 code, the user is only required to specify the independent and dependent variable names of the target differentiation and a differentiation starting point in the program. The $\mathrm{AD}$ tool then determines the variables that require derivative computations. It formulates the appropriate forward or reverse mode derivative expressions for these variables and generates new FORTRAN 77 code for the computation of both the original simulation and the associated derivatives. The ADIFOR-generated code is compiled (with special libraries) and executed like the original code.

The forward mode of differentiation was used to compute the $S \& C$ derivatives because generally, only a few input, or independent, variables are identified for differentiation within a given code generation (compared with 12 output, or dependent, variables). The reverse mode of AD may be employed to calculate the $\mathrm{S} \& \mathrm{C}$ derivatives for morphing vehicles, as in References 12 and 13, where the potentially thousands of independent variables (perhaps, each surface grid point, along with the $\mathbf{3 0}$ or so flight condition and orientation variables) would greatly outnumber the 12 dependent variables. 
Grid Resolution Studies

Three assumptions were made at the start of this work: 1) a panel method CFD code with a coarse surface grid could be used in conceptual or preliminary design whereas Euler / Navier-Stokes computations are likely to require so much execution time as to be incompatible with conceptual or preliminary design, 2) the surface grid shown in Figure 1 was probably too coarse to be accurate but represented a good starting point, and 3) grid resolution could be increased at any time, once the computational processes were established, to smoothly increase the accuracy of the results. It remains to be seen if the first assumption is valid. However, as illustrated in Figure 2 of the Results section of the paper, the second assumption is valid; this surface grid inaccurately approximates even the most basic of S\&C parameters, such as the lift variation with angle of attack, even at low angles of attack where a panel method formulation should be valid and vertical was not expected to affect the computation. This led the authors to, among other things, systematically examine the surface grid resolution requirements through a variety of studies, essentially testing the validity of the third assumption.

A considerable amount of processing and analysis was involved in obtaining the surface grids to be used for the current surface grid resolution studies, which were obtained as subsets of volume grids. The F-16XL volume grid history is as follows:

1. The first grid was a 30-block, structured, half-configuration volume grid containing 1,502,138 grid points (FINEST_VOL1); it was taken from previous Euler studies of References 39 and 43. Preliminary analysis using an earlier version of PMARC and an Euler/Navier-Stokes code was performed with this grid, but it provided unsuitable convergence for Navier-Stokes solutions in studies related to References 15 and 16 and was therefore abandoned.

2. A new 36-block, structured, full configuration volume grid containing 6,293,908 grid points (FINEST_VOL2) was developed for a modified F-16XL. An engine inlet fairing was added and tip missiles and launchers were removed. This configuration had improved grid resolution above the wing upper surface to enable users to capture votical flow over the upper wing surface with Euler/Navier-Stokes computations. Studies using this grid have not yet been performed.
3. The $Y-Z$ coordinate directions of the FINEST_VOL2 grid were swapped to conform with PMARC requirements.

4. Alternate grid points in each of the $\mathrm{I}-\mathrm{J}-\mathrm{K}$ coordinate directions were removed to produce another volume grid (MEDIUM_VOL, details presented in Table 1).

5. Again, alternate grid points in each of the I-J-K coordinate directions were removed to produce yet another volume grid (COARSE_VOL).

Surface grids consisting of 56 patches were extracted as subsets from each of the volume grids as follows:

1. A surface grid containing 113,856 grid points and 108,736 grid panels (FINEST_SRF) was obtained from the FINEST_VOL2 grid.

2. A reduced surface grid containing MEDIUM_SRF was obtained from the MEDIUM_VOL grid.

3. A further reduced surface grid (COARSE_SRF) was obtained from the COARSE_VOL grid.

4. Individual I-and J-grid lines from the COARSE_SRF grid were selected to be retained through two further sessions of coarsening (COARSE_SRF2 and COARSE_SRF3, respectively).

All the above surface grids required some I-J reordering of grid lines to conform with the PMARC geometry convention for "correct side out" patches. The majority of runs performed during this study used the COARSE_SRF3, I-J surface grid, or variations of this grid (described subsequently). Details about all the volume and surface grids are presented in Table 1.

Six types of surface grid resolution studies were conducted with the PMARC code. The studies used:

1. Externally generated grids for a symmetric, unswept, untapered, parabolic arc wing with an aspect ratio of two; alpha $=10$ degrees $(37$ cases executed).

2. Externally generated grids for configurations similar to the F-16XL wing plus fuselage carry-through (COARSE3_SRF) with parabolic arc wing sections; alpha $=10$ degrees $(45$ cases executed).

3. Internally generated grids based on the original F-16XL configuration (COARSE3_SRF) input to PMARC, modified through input choices to PMARC; alpha $=10$ degrees $(34$ cases executed). 
4. Externally generated grids starting from higher resolution surface geometries (FINEST_SRF, MEDIUM_SRF, and COARSE_SRF); alpha $=10$ degrees $(3$ cases executed or attempted).

5. Externally generated grids for configurations similar to the F-16XL wing plus part of the fuselage with parabolic arc wing sections; alpha $=\mathbf{3 0}$ degrees ( 22 cases executed).

6. Externally generated grids for configurations similar to the F-16XL wing plus part of the fuselage with parabolic arc wing sections; small amplitude forced pitch oscillation about alpha $=0$ degrees ( 30 cases executed).

The cases in group 4 failed to produce any useful results; cases in groups 5 and 6 produced results similar to the cases in group 2. Only the results from groups 1 , 2 , and 3 are reported in this paper. The surface and volume grid history/usage is summarized in Table 1.

\section{RESULTS}

As mentioned previously, Figure 1 shows a three-view of the computational geometry of the F-16XL used for most of these calculations; the grayscale indicate different surface patches in the PMARC geometry COARSE3_SRF input file. The surface resolution, consisting of 984 surface points and 566 surface panels, is moderately coarse by CFD standards. Results are first presented for the static forces and moments, then for dynamic situations. Figure 2 shows a comparison of computed lift, pitching moment, and normal force coefficient data with measured data. ${ }^{9.3}$ using this geometry. Circles in the figure are the lift force coefficient, triangles are the pitching moment coefficient, and squares are normal force coefficient; open symbols represent the computation and solid symbols are the data from Reference 9, as well as the solid diamonds representing the lift force coefficient from Reference 43 . The computed data reflects the general character of the measured data, but the computed data differs significantly with both sets of measured data. It was assumed that most of the differences in the lift and normal force coefficient computed/measured data comparisons could be attributed to: 1) at high angles of attack, the inability of the PMARC code to model the vortical and viscous flows, 2) uncertainty propagation due to uncertainties in the angle of attack, and 3) the surface resolution used in the PMARC computations for all angles of attack. Minor differences between the tested geometry and the computational geometry may also contribute to the differences in the forces and moments.

Vortex-generated lift is known to occur over the wing, ${ }^{39}$ and the PMARC code cannot model this flow feature. However, several altempts were made to simulate the effects of vortical flow by attaching additional wakes to the upper wing surface near the leading edge and/or over the mid-chord of the wing. Two such attempts are shown in Figure 3; the figure includes the same lift force coefficient data as in Figure 2 , but with two additional curves representing the attempts to add additional wake to the wing upper surface, indicated with dashed lines. Indeed, the data comparisons could be improved at high angles of attack ( 30 to 50 degrees) by adding wakes separating from the mid-chord of the wing. However, such results required knowing either where the separation might occur or what value of the lift or normal force coefficient was to be obtained. The same technique significantly overpredicted the lift and normal force coefficient at 10 to 20 degrees angle of attack, indicated by the dashed curves extending off the top of the figure. This technique would need to be tailored for specific flow conditions. The cases should be repeated with both Euler and Navier-Stokes codes to truly assess the impact of vortex formation and bursting.

The effect of uncertainty propagation through the CFD code was also examined; the same cases shown in Figure 2 were executed with an ADIFORgenerated Hessian (second derivative) code. For this case, derivatives of the forces and moments and their first derivatives with respect to angle of attack were differentiated again with respect to angle of attack. Uncertainties with respect to the angle of attack were computed and propagated through the code, proportional to variable gradients with respect to the uncertain variable, via Equation 1. The results from this study are shown as the error bars in Figures 3 and 4 and in Table 2. Figure 3 shows the computed lift coefficient data of Figure 2, with added error bars showing the uncertainty in $C_{L}$ due to a one-sigma uncertainty (for illustration purposes) in the angle of attack (AOA) for an assumed uncertainty in the measured angle of attack given by $\sigma_{A O A}=0.1$ degrees, determined from the measured data. Figure 4 shows the lift curve slope. $C_{L \alpha}$, for the data in Figure 2, with added error bars showing uncertainty due to a onesigma uncertainty in the angle of attack. Figures 3 and 4 indicate that the propagated uncertainties due to uncertainties in angle of attack account for some, but only a small part, of the differences in the computed and measured lift coefficient. The data shown in Table 2 illustrates a two-variable (angle of attack and pitch rate, $Q$ ) uncertainty assessment. The table shows the variable name, the uncertain variable name, the computed output variable sigma (from Eq. 1), and one-, two-, and three-sigma (representing 84.1, 97.7, and $99.9 \%$, respectively) low/high uncertainty bounds for each variable, as well as the mean value for each 
variable. Table 2 illustrates a typical preliminary uncertainty analysis for the F-16XL configuration. The uncertainties in $C_{L}, \frac{d C_{L}}{d \alpha}$, and $\frac{d C_{L}}{d q}$ (with $\alpha$ in degrees and $q$ in degrees per second) are presented as calculated from first and second derivatives of $C_{L}$ with respect to $\alpha$ and $q$, assuming $\sigma_{\text {inpur }}=0.1$ for both $\alpha$ and $q$. The same capability is already available for all of the forces and moments in both the wind and body axis systems; results can be easily computed for any of the independent variabies of interest.

The final element contributing to the discrepancy between measured and computed data in Figure 2 was the effect of surface resolution. A series of grid resolution studies, described previously, was performed. The results of these studies are shown in Figures 5 through 10. Figure 5 shows an envelope of computed lift coefficient data of various fidelities for a symmetric, unswept, untapered, parabolic arc wing with an aspect ratio of two, where alpha $=10$ degrees (group 1 grid resolutions studies) at various surface resolutions, plotted as a function of the $\log 10(\log$ base 10) of the number of surface panels. The envelope of solutions includes data from 37 cases (group 1 grid resolution studies) with the total surface (full span) resolution ranging from 8 to 4096 panels increasing in even multiples of 2 , with distributions ranging from 2 to 256 panels chordwise, and 2 to 64 panels spanwise, although not all possible combinations within that range were executed due to time and memory limitations on the available computers. Also shown in Figure 5 is an analytic computation of the lift coefficient taken from Reference 2. The envelope of computed lift coefficient data demonstrates grid independence at dense surface resolutions (thousands of surface panels); the data become both independent of the total number of surface panels and their distributions spanwise and chordwise, as well as agreeing well with the analytic solution. These results provide great evidence that the PMARC code can produce useful results for some geometries and flight conditions at sufficiently dense surface resolutions.

Figure 6 illustrates the envelopes of data for the drag and the pitching moment coefficients. Similar to Figure 5, grid independence is observed in drag coefficient. The apparent lack of grid independence at dense surface resolutions in the pitching moment is simply an artifact of the magnified vertical scale compared with Figure 5. Nearly the same level of grid independence is achieved for both the lift force and pitching moment coefficient data. The convergence achieved in the drag coefficient is actually two orders of magnitude greater than either the lift force or pitching moment coefficient. Figure 7 shows the same lift force coefficient data as in Figure 5, but plotted with curves of constant chordwise resolution (varying spanwise resolution) around the airfoil section. Comparing Figures 5 and 7, the minimum lift force boundary in Figure 5 is simply a composite of all the solutions with only four panels chordwise. In contrast, the maximum boundary in Figure 5 is a composite of the leftmost points on the curves in Figure 7 (those point with minimum spanwise resolution for a given chordwise resolution). A similar but inverse behavior was observed for the pitching moment. These results suggest, as expected, that four panels chordwise (around the airfoil) are too few; the resulting wing sections are modeled only as a composite of forward and rearward facing wedges, resulting in large errors in the predicted forces and moments. More panels chordwise appear to smoothly and significantly improve the fidelity of the PMARC solution. Put another way, the results only with only four panels chordwise never asymptotically approach the expected value with increased surface resolution for the cases studied. A discontinuous approach to the analytic solution is observed; the surface grid resolution must be "good enough" to begin an asymptotic approach to analytic value. A similar conclusion was reported in Reference 37. The F-16XL grid used for most of the studies has 10 panels chordwise around the airfoil section and 8 panels spanwise for each wing semispan; Based upon this result alone, one might expect the grid shown in Figure 1 to be sufficient to accurately approximate the measured results, which not the case. Figure 8 shows an envelope of lift coefficient data for wing shapes similar to the F-16XL planform (group 2 grid resolution studies, 45 cases). Behavior similar to that seen in Figure 5 is observed, but grid independence is not achieved to the same degree. The drag force and pitching moment data envelopes for these cases are similar to those of Figures 6 . More panels, above a certain minimum threshold chordwise, improve the accuracy of the computed data. However, an important limiting effect in accuracy due to the more complex F-16XL wing geometry, compared to the swept tapered wing of Figures 5-7, is observed in Figure 8.

Figure 9 shows a scatter plot (rather than an envelope of data) for the 34 cases of group 3 grid resolution studies. In this study, the total number of surface panels ranged from 504 to 2486 , with the nominal grid containing 566 panels. The distribution of the panels varied from run to run, with surface resolution increasing either over the whole vehicle, only the wings, or select regions of the wings. This was a systematic study performed with the goal of matching the lift coefficient at 10 degrees angle of attack from 
Reference 9. Many executions beyond those 34 reported here were performed that resulted in true "outlier solutions" with unreasonably large lift coefficients (greater than 2.0); the same can also be said of the previous two grid resolution studies, although more "outlier solutions" occurred is the studies associated with Figure 9. Also shown in Figure 9 are the values of the lift coefficient obtained from References 9 and 54. Most of the computed results in Figure 9 fall outside of the range of the two reference solutions, and no grid independence, nor any approach to the expected value, is discernable. Taken with previous results, this suggests that surface grid smoothness, in addition to the surface grid resolution and distribution, plays a big role in the accuracy of the PMARC results for lift coefficient. In contrast, as shown in Figure 10, nearly any grid distribution or resolution may predict the expected pitching moment for this flow condition to acceptable accuracy.

To summarize, several important effects have been identified in Figures 5-10 which strongly affect the accuracy of the PMARC results: 1) the total grid density, 2) the grid distribution spanwise and chordwise, 3) minimum thresholds of surface resolution may exist for obtaining accurate results, 4) configuration geometric complexity, and 5) grid smoothness. It is important to realize that once a grid has been obtained for a given configuration within a given study, the user may have little, if any, means to improve upon any of these aspects of the grid, which significantly affect the quality of the results. At this point, it is assumed that a grid of sufficient quality can be obtained and validated for use. The remainder of the figures illustrate in a qualitative sense what could be done with such a grid for $\mathrm{S} \& \mathrm{C}$ applications.

Figure 11 shows the original vehicle geometry, with wake trailing from the top of the vertical tail, during a body axis roll maneuver. For all computations, other wakes (not shown in Figure 11) were also attached to the trailing edges of both wings. Figure 12 shows the vehicle, again with wake trailing from the top of the vertical tail, during a forced oscillation in roll maneuver. The reader should note the two reversals in rotary direction illustrated by the wake. In this case, the only useful "pure rotary" portion of the motion occurs between the two reversals after start-up transients have diminished; this is typical of how the maneuver might be performed in a wind tunnel test where the facility does not have "slip ring" capability. CFD offers the ability to perform the roll maneuver, without any support or wall interference effects, in either the pure rotary or in forced oscillation modes.

Figures 13 and 14 illustrate two advanced experimental techniques used to determine dynamic S\&C derivatives. Figure 13 shows a coning motion; the body rotates about the velocity vector, which is aligned with oncoming flow stream. Figure 14 illustrates an oscillatory coning (or, inclined axis roll (IAR)) motion where the body rotates around an axis not aligned with the velocity vector. The motion is named for the oscillatory behavior of the angles of attack and sideslip observed during the motion. These types of motions can only be performed in a few wind and water tunnels globally. Figures 15 and 16 illustrate the CFD simulation of these two coning motions. In Figure 15, the original geometry is rotated to an initial pitch orientation angle, here 30.8 degrees, before the CFD solution is performed. A body axis roll maneuver is then performed with the rotated geometry and with the velocity vector aligned with the rotational vector, as above in Figure 11. The motion could also be performed with an unrotated body and imposed angle of attack, but the body-axis forces differ between the two scenarios. In Figure 16, an angle of attack of 50 degrees has been imposed on the body axis roll of the rotated geometry illustrated in Figure 15. The angle of attack in Figure 16, roll rates, and the forward speeds of Figures 11, 12, 15, and 16 were chosen simply to illustrate the maneuvers and do not reflect actual tunnel test conditions, although the body rotation angle of 30.8 was chosen to match experimental data available for the F-16XL tested in the NASA LaRC 14- by 22Foot Subsonic Tunnel. Figures 15 and 16 are included simply to illustrate the ease with which advanced testing techniques can be simulated through CFD. Obviously, the maneuvers should be performed with a grid of sufficient density, using guidance obtained from the above grid resolutions studies, and with a code that is able to capture all the relevant flow physics.

Typical execution times for the F-16XL geometry in the original PMARC code are about 3.25 minutes each on a R 12000 dual processor of a Silicon Graphics ${ }^{\circledR}$ Octane ${ }^{\circledR}$. The forward mode ADIFORgenerated PMARC aerodynamic analysis, including the computation of angle of attack and sideslip derivatives, required approximately 6.29 minutes for the same single-processor execution scheme. The second derivative calculation, required for the uncertainty propagation analysis, required for the same two independent variables required about 14.10 minutes. All the calculations were performed in 64-bit arithmetic. The RAM requirements were about 130 MB for the PMARC function and $213 \mathrm{MB}$ for the function plus its derivatives with respect to angle of attack and angle of sideslip. The second derivative code required about $481 \mathrm{MB}$ of storage. Late in the studies, the PMARC code was found to execute three to five times faster per case on a PC cluster, composed of a host and 32 worker nodes. Each worker node uses a 2193 Mhz central processing unit (CPU), the Linux 
2.4.18 operating system, and a Lahey/Fujitsu

Optimizing Fortran 95 compiler.

\section{CONCLUSIONS}

The Panel Method Ames Research Center (PMARC) code, version 14.10, has been differentiated in several versions to provide exact first and second derivatives of the forces and moments with respect to wide variety of inputs. The original and differentiated codes have been executed to simulate a variety of motions typically used in experimental facilities to obtain dynamic stability and control (S\&C) derivatives. Initiai results, including input uncertainty propagation error bounds, using an F-16XL configuration as the test vehicle were shown. The results were obtained with a coarse surface grid resolution computational model using reasonable amounts of computer time and memory. The results generally reflect the trends of the measured F-16XL data, but the lift and normal force coefficient data were substantially different from the measured data.

Additional wakes separating from the mid chord upper surface region of the wing could be used to improve the accuracy of the computed results, but this technique requires some knowledge about the expected flowfield characteristics. Propagated uncertainty bounds were also shown to account for a small part of the difference between the computed and measured data at low angles of attack. In addition, the results of numerous surface grid resolution studies were reported. The surface grid resolution studies show that a minimum of eight panels chordwise around the airfoil sections of a wing are necessary to obtain meaningful approximations to the expected forces and moments. The results are strongly influenced by the surface resolution in both the chordwise and spanwise directions, as well as the geometric complexity of the body and the smoothness of the grid distribution. For smooth distributions of surface panels, grid independence of results and agreement with known analytic solutions can be obtained with sufficiently dense surface resolutions. In the absence of smooth surface resolution, grid independence of the results cannot be achieved; in fact, the results in such cases do not appear to even converge to common values.

Finally, the work reported here demonstrates some potential for using very coarse computational models to predict $S \& C$ characteristics of complex vehicles, especially when measured data is available to calibrate the computations. Such coarse computational models may be useful in the conceptual or preliminary design arena, but they represent only the first phase of applying CFD codes to aircraft S\&C problems. The work also demonstrated several computational techniques that can be used to further the application of CFD to stability and control applications.

\section{REFERENCES}

1. Nelson, Robert C., Flight Stability and Automatic Control (Second Edition), McGraw Hill International Editions Aerospace Science \& Technology Series, New York, New York, 1998.

2. Nicolai, Leland M., Fundamentals of Aircraft Design, METS, Inc. Xenia, Ohio, 1975.

3. Roskam, Jan, Airplane Flight Dynamics and Automatic Flight Controls, Parts I and II, DARcorporation, Lawrence, Kansas, 2001.

4. Roskam, Jan, Airplane Design, Part VII:

$\bar{D}$ etermination of Stability, Control, and

Performance Characteristics: FAR and Military

Requirements, DARcorporation, Lawrence, Kansas, 2002.

5. Roskam, Jan, Advanced Aircraft Analysis (Software Package and Documentation), DARcorporation, Lawrence, Kansas, 2001.

6. McDonnell Douglas Astronautics Company, St. Louis Division, The USAF Stability and Control DATCOM, Volume I, Users Manual, McDonnell Douglas Astronautics Company, St. Louis Division, St. Louis, Missouri, 1979.

7. Lan, Edward C., "VORSTAB - A Computer Program for Calculating Lateral-Directional Stability Derivatives with Vortex Flow Effect," NASA Contractor Report 172501, January 1987.

8. Tavoularis, S., "Equivalence Between Sideslip and Roll In Wind-Tunnel Model Testing," AIAA J. Aircraft. Vol. 36, No. 5, pages 895-896, May, 1999.

9. Klein, V., Murphy, P. C., Curry, T. J., and Brandon, J. M., "Analysis of Wind Tunnel Longitudinal Static and Oscillatory Data of the F16XL Aircraft," NASA/TM-97-206276.

10. Green, Lawrence L., Spence, A. M., and Murphy, P. C.: Computational Methods for Dynamic Stability and Control Derivatives. AIAA 2004-0015, January 5-8, 2004.

11. Dorsett, K. M., and Mehl, D. R., "Innovative Control Effectors (ICE)," Wright Laboratory Report, WL-TR-96-3043, Jan. 1996.

12. Raney, D. L., Montgomery, R. C., and Green, L. L., and Park, M. A., "Flight Control Using Distributed Shape-Change Effector Arrays," AIAA 2000-1560, April 2000.

13. Park, M., Green, L., Montgomery, R., and Raney, D., "Determination of Stability and Control Derivatives Using Computational Fluid Dynamics and Automatic Differentiation," AIAA paper 99-3136, June 1999.

14. Limache, A. C., and Cliff, E. M., "Aerodynamic Sensitivity Theory for Rotary Stability Derivatives," AIAA Paper 98-4313, Aug. 1998. 
15. Park, Michacl A. and Green, Lawrence L., "Steady-State Computation of Constant Rotational Rate Dynamic Stability Derivatives", AIAA 200-4321. August 2000.

16. Park, Michacl A., Determination of Static and Dynamic Stability and Control Derivatives With Computational Fluid Dynamics and Automatic Differentiation, Master Thesis, The George Washington University, August 2000.

17. Gainer, Thomas G., "A Discrete-Vortex Method for Studying the Wing Rock of Delta Wings," NASA/TP-2002-211965, December 2002.

18. Melville, Reid. "Aeroelastic Instability of Tactical Aircraft in Nonlinear Flow Regeims," AIAA 2002-2970, June, 2002.

19. Jouannet, C. and Krus, P., "Lift Coefficient Predictions for Delta Wing Under Pitching Motions," AIAA 2002-2969, June 2002.

20. Kay, Jacob, "Acquiring and Modeling Unsteady Aerodynaimc Characteristics," AIAA 2000-3907, August 2000.

21. Katz, Joseph, and Schiff, Lewis B., "Modeling Aerodynamic Responses to Aircraft Maneuvers-A numerical Validation," AIAA J. Aircraft, Vol. 23, No. 1, January 1986.

22. van Dam, C. P., DalBello, T., and Chao, D. D., "Prediction of Flows About Forebodies at High-Angle-of-Attack Dynamic Conditions," Final Report of NASA Langley Research Center Grant NAG-1-2006, May 2000.

23. Youngren, H. H., Bouchard, E. E., Coopersmith, R. M., and Miranda, L. R., "Comparison of Panel Method Formulations and its Influence on the Development of QUADPAN, an Advanced Low Order Method," AIAA Paper 83-1827, July 1983.

24. Miranda. L. R., Elliott, R. D., and Baker, W. M., "A Generalized Vortex Lattice Method of Subsonic and Supersonic Flow Applications," NASA CR-2865, Dec. 1977.

25. Simon, J. M., "Dynamic Derivative Data for High-Angle-of-Attack Simulation," AIAA paper 92-4355, Aug. 1992.

26. Blake, W. B., Dixon, C. J., and Adler, C. O., "Development of a High-Angle-of-Attack Stability and Control Prediction Code," AIAA Paper 92-4354, Aug. 1992.

27. Ahn, T., Kim, C., Rho, O. H., and Kim, H.-J., "Dynamic Stall Control Using Aerodynamic Sensitivity Analysis," AIAA 2001-0255, January 2001.

28. 1988 Report to the Aerospace Profession XXXIII, Thirty-Third Symposium Proceedings, September 1989.
29. Albright. A. E., Dixon, C. J., Hegedus, M. C., "Modification and Validation of Conceptual Design Aerodynamic Prediction Method HASC95 With VTXCHN," NASA CR-4712, Mar. 1996.

30. Brandon, J.M., and J.V. Foster: Recent Dynamic Measurements and Considerations for Aerodynamic Modeling of Fighter Airplane Configurations, AIAA 98-4447, August 1998.

31. Klein, V., and Noderer, K.D.: Modeling of Aircraft Unsteady Aerodynamic Characteristics", Part I - Postulated Models, NASA TM 109120, May, 1994; Part 2 - Parameters Estimated From Wind Tunnel Data, NASA TM 110161 , April 1995; Part 3 - Parameters Estimated From Flight Data, NASA TM 110259, May 1996.

32. Anderson, W. K., Newman, J. C., Whitfield, D. L., and Nielsen, E. J.: Sensitivity Analysis for the Navier-Stokes Equations on Unstructured Meshes Using Complex Variables". AIAA paper 99-3294, 1999.

33. Vatsa, V. N.: Computation of Sensitivity Derviatives of Navier-Stokes Equations Using Complex Variables, Fifth Symposium on the Large-Scale Analysis and Design and ISE, Williamsburg, Virginia, Oct. 12-15, 1999.

34. Hemsch, M., "Statistical Analysis of CFD Solutions from the Drag Prediction Workshop," AIAA 2002-0842, January 2002.

35. Hosder, S., Grossman, B., Haftka, R. T., Mason, W. H., and Watson, L. T., Observations on CFD Simulation Uncertainties, AIAA 20025531, September 2002.

36. Green, L., Lin, H.-Z., and Khalessi, M., "Probabilistic Methods for Uncertainty Propagation Applied to Aircraft Design,", AIAA Paper 2002-3140, June 2002.

37. Eca, L., Vaz, G. B., and Falco de Campos, A. C., "Verfication Study of Low and Higher-Order Potential Based Panel Methods for 2D Foils," AIAA 2002-3112, June 2002.

38. Putko, M. M., Taylor, III, A. C., Newman, P. A., and Green, L. L., "Approach for Input Uncertainty Propagation and Robust Design in CFD Using Sensitivity Derivatives," Journal of Fluids Engineering, published by the Society of Mechanical Engineers, Vol. 124, No. 1, March 2002, pp. 60-69 (see also.AIAA Paper 20012528, June 2001).

39. Lessard, Wendy B., "Subsonic Analysis of 0.04-Scale F-16XL Models Using an Unstructured Euler Code," NASA Technical Paper 3597, October 1996. 
40. Bischof, C., Carle, A.. Corliss, G., Griewank. A., and Hovland, P.. "ADIFOR-Generating Derivative Codes from Fortran Programs," Scientific Programming, No. 1, 1992, pp. 1-29.

41. Bischof, C., Carle, A., Khademi, P., and Mauer, A., "Automatic Differentiation of FORTRAN," IEEE Computational Science \& Engineering, Fall 1996.

42. Ashby, Dale L., Dudley, Michael R., Iguchi, Steve K., Browne, Lindsey, and Katz, Joseph, "Potential Flow Theory and Operation Guide for the Panel Code PMARC," NASA TM 102851. January $1999 \mathrm{i}$. (Ũnofíciailiy revised in 1999 for PMARC version 14).
43. Hahne, David E, "Low-Speed Acrodynamic Data for an 0.18-Scale Model of an F-16XL With Various Leading-Edge Modifications," NASA/TM-1999-209703, December 1999.

44. Kramer, Brian R.: Experimental Evaluation of Superposition Techniques Applied to Dynamic Aerodynamics. AIAA 2002-0700, January 14-17, 2002.

45. Eidetics Corporation: Aircraft Dynamics: Nonlinear Math Modeling. Annual Report June 2002. Contract NAS1-01091.

46. Carle, A. and Fagan, M., "Overview of Adifor 3.0." Department of Computational and Applied Mathematics, Rice University, CAAMTR 00-02, Jan. 2000. 
Table 1 Summary of F-16XL geometries, grids, and grid usages.

\begin{tabular}{|c|c|c|c|c|}
\hline Grid & Description & Grid Size & Surface Resolution & Grid Usage \\
\hline FINEST_VOL1 & $\begin{array}{l}\text { Half configuration } \\
\text { volume grid w/tip } \\
\text { missiles \& launchers }\end{array}$ & $\begin{array}{c}30 \text { blocks } \\
1,502.138 \text { grid } \\
\text { points }\end{array}$ & Not applicable & $\begin{array}{l}\text { Not used for the } \\
\text { current studies }\end{array}$ \\
\hline FINEST_VOL2 & $\begin{array}{l}\text { Full configuration } \\
\text { volume grid w/inlet } \\
\text { fairing }\end{array}$ & $\begin{array}{c}36 \text { blocks } \\
6,293,908 \text { grid } \\
\text { points }\end{array}$ & Not applicable & $\begin{array}{l}\text { Future Euler and } \\
\text { Navier-Stokes } \\
\text { code use planned }\end{array}$ \\
\hline MEDIUM_VOL & $\begin{array}{c}\text { Subset of } \\
\text { FINEST_VOL2 grid }\end{array}$ & $\begin{array}{c}36 \text { blocks } \\
837,924 \text { grid points }\end{array}$ & Not applicable & $\begin{array}{l}\text { Future Euler and } \\
\text { Navier-Stokes } \\
\text { code use planned }\end{array}$ \\
\hline COARSE_VOL & $\begin{array}{l}\text { Subset of } \\
\text { MEDIUM_VOL } \\
\text { grid }\end{array}$ & $\begin{array}{c}36 \text { blocks } \\
118,216 \text { grid points }\end{array}$ & Not applicable & $\begin{array}{l}\text { Future Euler and } \\
\text { Navier-Stokes } \\
\text { code use planned }\end{array}$ \\
\hline FINEST_SRF & $\begin{array}{c}\text { Surface grid } \\
\text { extracted from } \\
\text { FINEST_VOL2 } \\
\text { volume grid }\end{array}$ & $\begin{array}{c}56 \text { patches } \\
113,856 \text { grid points }\end{array}$ & $108,7.36$ surface panels & $\begin{array}{c}\text { Execution } \\
\text { attempted, } \\
\text { exceeded } \\
\text { available RAM }\end{array}$ \\
\hline MEDIUM_SRF & $\begin{array}{l}\text { Surface grid } \\
\text { extracted from } \\
\text { MEDIUM_VOL } \\
\text { volume grid }\end{array}$ & $\begin{array}{c}56 \text { patches } \\
29,772 \text { grid points }\end{array}$ & 27,184 surface panels & $\begin{array}{c}\text { Execution } \\
\text { attempted, } \\
\text { exceeded } \\
\text { available disk } \\
\text { space }\end{array}$ \\
\hline COARSE_SRF1 & $\begin{array}{l}\text { Surface grid } \\
\text { extracted from } \\
\text { COARSE_VOL } \\
\text { volume grid }\end{array}$ & $\begin{array}{c}56 \text { patches } \\
8,118 \text { grid points }\end{array}$ & 6,796 surface panels & $\begin{array}{c}\text { Execution } \\
\text { completed, } \\
\text { unreasonably } \\
\text { large forces and } \\
\text { moments } \\
\text { computed } \\
\end{array}$ \\
\hline COARSE_SRF2 & $\begin{array}{c}\text { Surface grid } \\
\text { extracted from } \\
\text { COARSE_VOL } \\
\text { volume grid }\end{array}$ & $\begin{array}{c}56 \text { patches } \\
2,434 \text { grid points }\end{array}$ & 1,744 surface panels & $\begin{array}{l}\text { Not used for the } \\
\text { current studies }\end{array}$ \\
\hline COARSE_SRF3 & $\begin{array}{c}\text { Surface grid } \\
\text { extracted from } \\
\text { COARSE_VOL } \\
\text { volume grid }\end{array}$ & $\begin{array}{c}56 \text { patches } \\
984 \text { grid points }\end{array}$ & 566 surface panels & $\begin{array}{l}\text { Used extensively } \\
\text { for the current } \\
\text { studies }\end{array}$ \\
\hline
\end{tabular}

Table 2 Sample uncertainty analysis for F-16XL all values of $\sigma_{\text {input }}=0.1$.

\begin{tabular}{|c|c|c|c|c|c|c|c|c|c|}
\hline Variable Name & $\begin{array}{c}\text { Uncertainty } \\
\text { Variable }\end{array}$ & $\begin{array}{c}\text { Computed } \\
\text { sigma }\end{array}$ & $\begin{array}{c}\mathbf{9 9 . 9 \%} \\
\text { Low }\end{array}$ & $\begin{array}{c}\mathbf{9 7 . 7 \%} \\
\text { Low }\end{array}$ & $\begin{array}{c}\mathbf{8 4 . 1 \%} \\
\text { Low }\end{array}$ & $\begin{array}{c}\text { Mean } \\
\text { Value }\end{array}$ & $\begin{array}{c}\mathbf{8 4 . 1 \%} \\
\text { High }\end{array}$ & $\begin{array}{c}\mathbf{9 7 . 7 \%} \\
\text { High }\end{array}$ & $\begin{array}{c}\mathbf{9 9 . 9 \%} \\
\text { High }\end{array}$ \\
\hline dCL/dALPDEG & ALPDEG & $4.72 \mathrm{E}-05$ & $2.02 \mathrm{E}-02$ & $2.03 \mathrm{E}-02$ & $2.03 \mathrm{E}-02$ & $2.04 \mathrm{E}-02$ & $2.04 \mathrm{E}-02$ & $2.05 \mathrm{E}-02$ & $2.05 \mathrm{E}-02$ \\
\hline $\mathrm{dCL} / \mathrm{d}$ ALPDEG & $\mathrm{Q}$ & $4.97 \mathrm{E}-05$ & $2.02 \mathrm{E}-02$ & $2.03 \mathrm{E}-02$ & $2.03 \mathrm{E}-02$ & $2.04 \mathrm{E}-02$ & $2.04 \mathrm{E}-02$ & $2.05 \mathrm{E}-02$ & $2.05 \mathrm{E}-02$ \\
\hline $\mathrm{dCL} \mathrm{dALPDEG}$ & ALPDEG $+\mathrm{Q}$ & $6.85 \mathrm{E}-05$ & $2.02 \mathrm{E}-02$ & $2.02 \mathrm{E}-02$ & $2.03 \mathrm{E}-02$ & $2.04 \mathrm{E}-02$ & $2.04 \mathrm{E}-02$ & $2.05 \mathrm{E}-02$ & $2.06 \mathrm{E}-02$ \\
\hline $\mathrm{CL}$ & ALPDEG & $2.04 \mathrm{E}-03$ & $3.95 \mathrm{E}-01$ & $3.97 \mathrm{E}-01$ & $3.99 \mathrm{E}-01$ & $4.01 \mathrm{E}-01$ & $4.03 \mathrm{E}-01$ & $4.05 \mathrm{E}-01$ & $4.07 \mathrm{E}-01$ \\
\hline $\mathrm{dCL} / \mathrm{dQ}$ & ALPDEG & $4.97 \mathrm{E}-05$ & $2.39 \mathrm{E}-02$ & $2.40 \mathrm{E}-02$ & $2.40 \mathrm{E}-02$ & $2.41 \mathrm{E}-02$ & $2.41 \mathrm{E}-02$ & $2.42 \mathrm{E}-02$ & $2.42 \mathrm{E}-02$ \\
\hline $\mathrm{dCL} / \mathrm{dQ}$ & $\mathrm{Q}$ & $1.23 \mathrm{E}-03$ & $2.04 \mathrm{E}-02$ & $2.16 \mathrm{E}-02$ & $2.28 \mathrm{E}-02$ & $2.41 \mathrm{E}-02$ & $2.53 \mathrm{E}-02$ & $2.65 \mathrm{E}-02$ & $2.78 \mathrm{E}-02$ \\
\hline $\mathrm{dCL} / \mathrm{dQ}$ & ALPDEG $\mathrm{Q}$ & $1.23 \mathrm{E}-03$ & $2.04 \mathrm{E}-02$ & $2.16 \mathrm{E}-02$ & $2.28 \mathrm{E}-02$ & $2.41 \mathrm{E}-02$ & $2.53 \mathrm{E}-02$ & $2.65 \mathrm{E}-02$ & $2.78 \mathrm{E}-02$ \\
\hline $\mathrm{CL}$ & ALPDEG & $2.41 \mathrm{E}-03$ & $3.94 \mathrm{E}-01$ & $3.96 \mathrm{E}-01$ & $3.98 \mathrm{E}-01$ & $4.01 \mathrm{E}-01$ & $4.03 \mathrm{E}-01$ & $4.06 \mathrm{E}-01$ & $4.08 \mathrm{E}-01$ \\
\hline $\mathrm{CL}$ & TOTAL & $3.15 \mathrm{E}-03$ & $3.91 \mathrm{E}-01$ & $3.95 \mathrm{E}-01$ & $3.98 \mathrm{E}-01$ & $4.01 \mathrm{E}-01$ & $4.04 \mathrm{E}-01$ & $4.07 \mathrm{E}-01$ & $4.10 \mathrm{E}-01$ \\
\hline
\end{tabular}



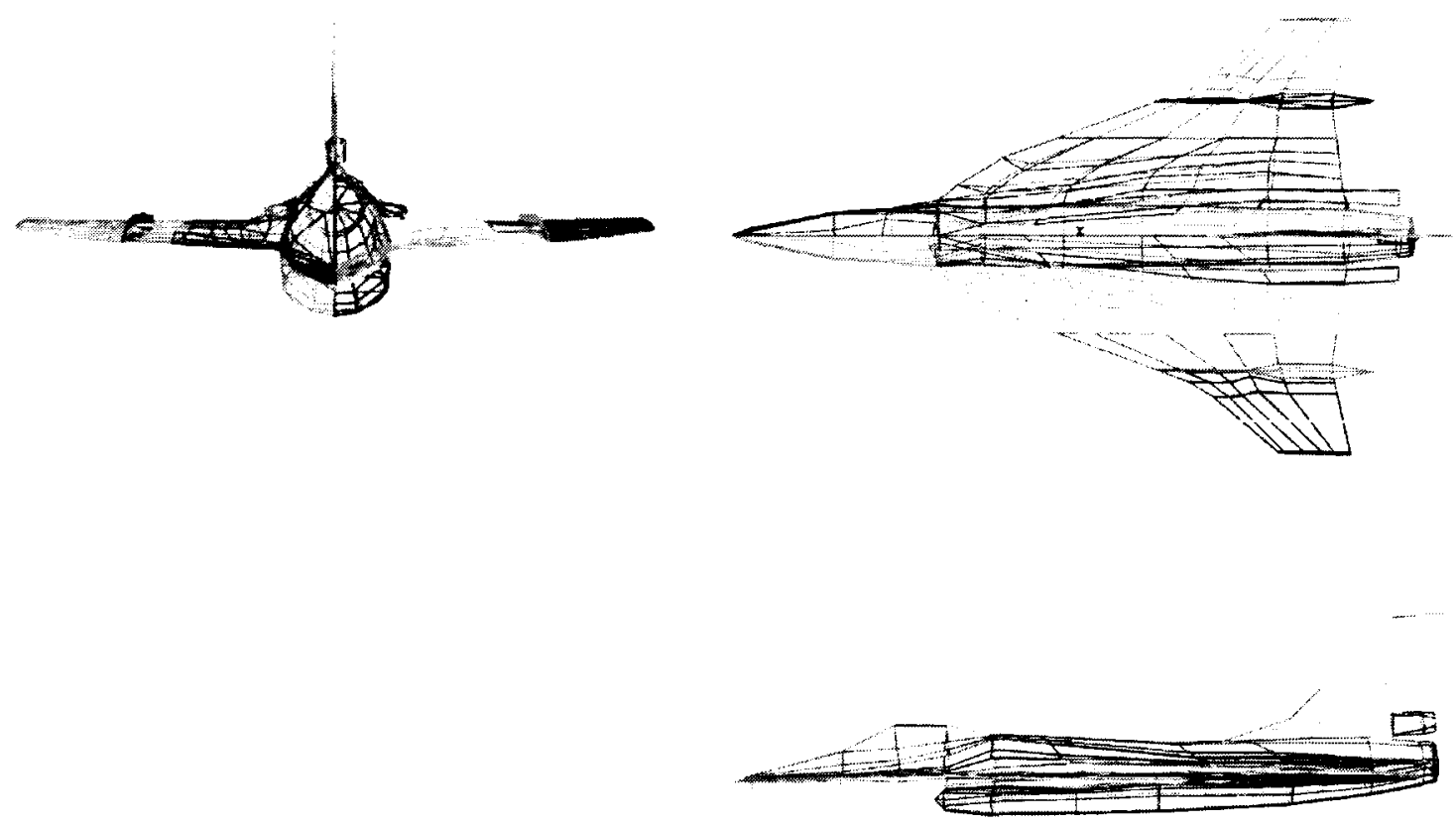

Figure 1. Three-view of F-16XL computational model. 


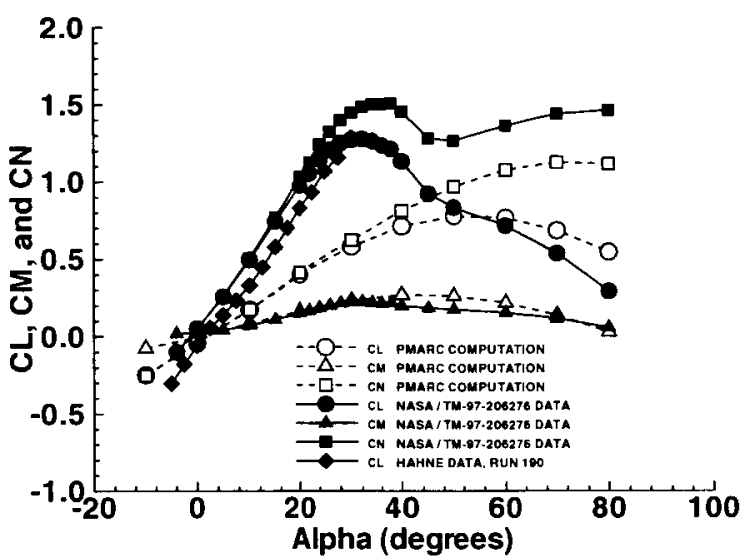

Figure 2. Computed static lift. moment, and normal force coefficient data and measured data.

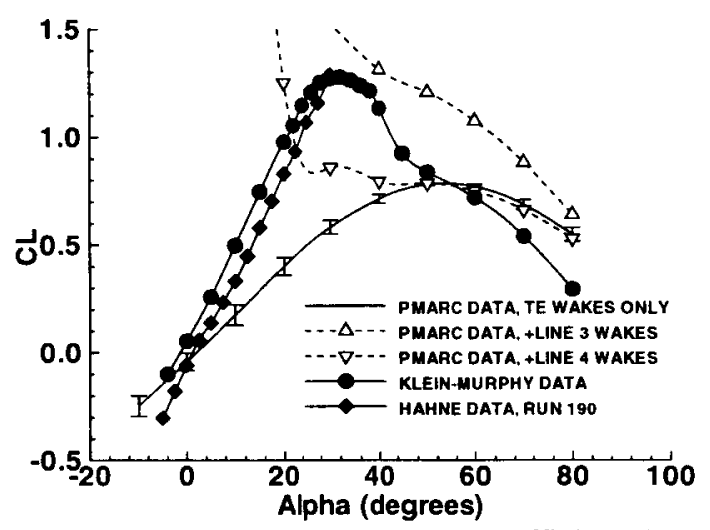

Figure 3. Computed static lift coefficient data, including uncertainty propagation error bars, and measured data.

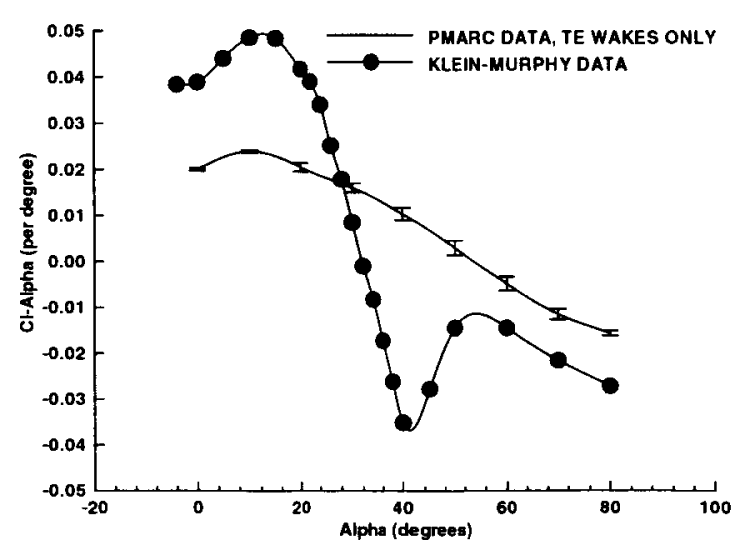

Figure 4. Computed static lift curve slope, $C_{L \alpha}$, coefficient data, including uncertainty propagation error bars, and measured data.

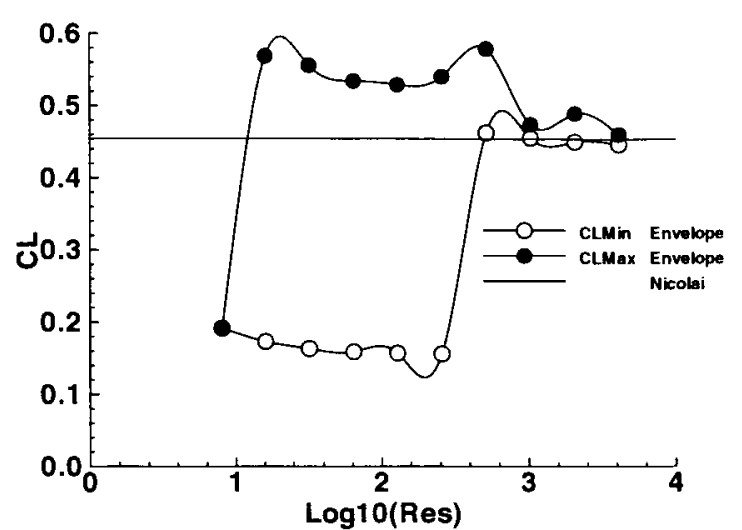

Figure 5. Envelope of computed lift coefficient data at various surface resolutions for $A R=2$, symmetric, parabolic wing with analytic data.

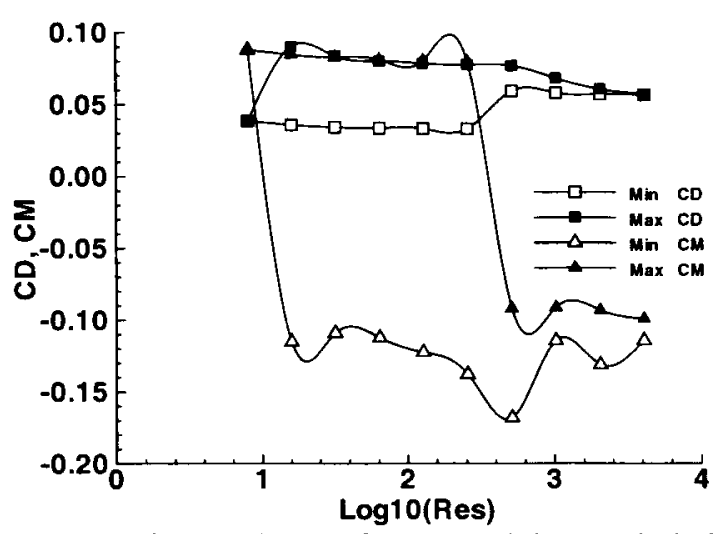

Figure 6. Envelopes of computed drag and pitching moment coefficient data at various surface resolutions, for $\mathrm{AR}=2$, symmetric, parabolic wing.

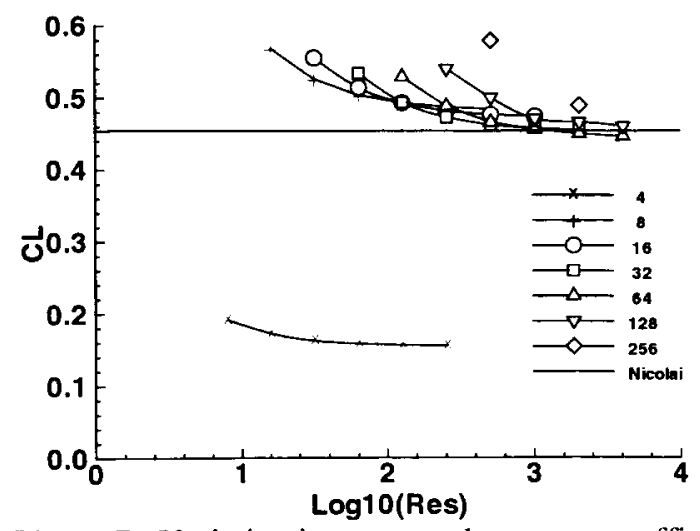

Figure 7. Variation in computed moment coefficient with surface resolution for $A R=2$, symmetric, parabolic wing and fixed chordwise resolutions. 


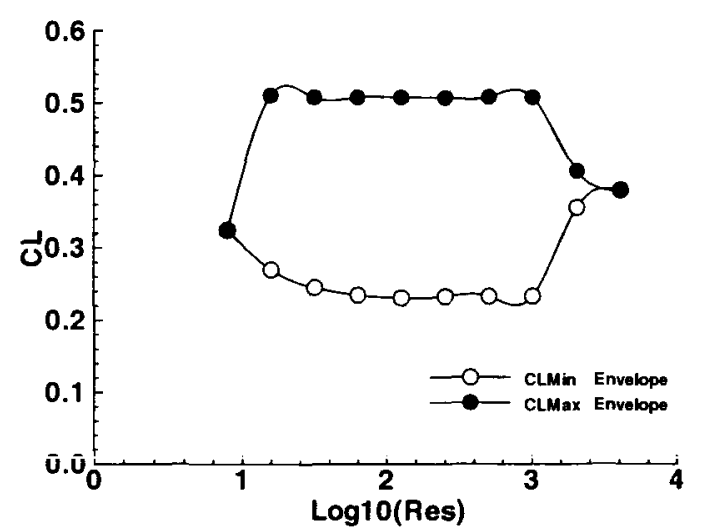

Figure 8. Envelope of computed lift coefficient data at various surface resolutions for configurations similar to F-16XL.

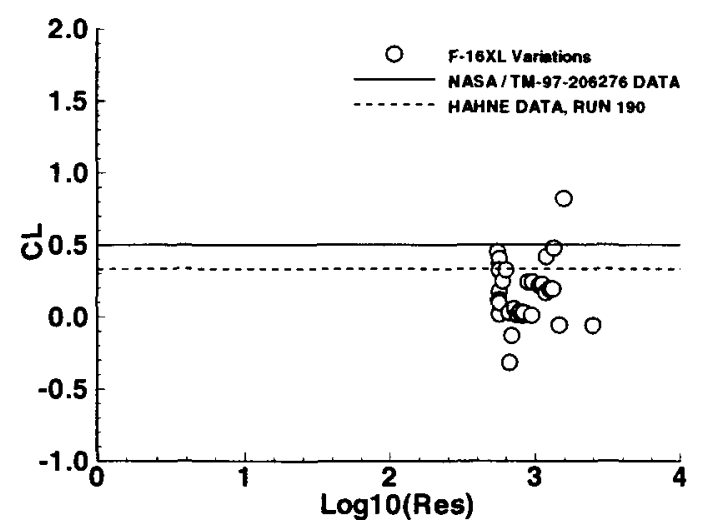

Figure 9. Variation in computed lift coefficient with surface resolution for the F-16XL configuration.

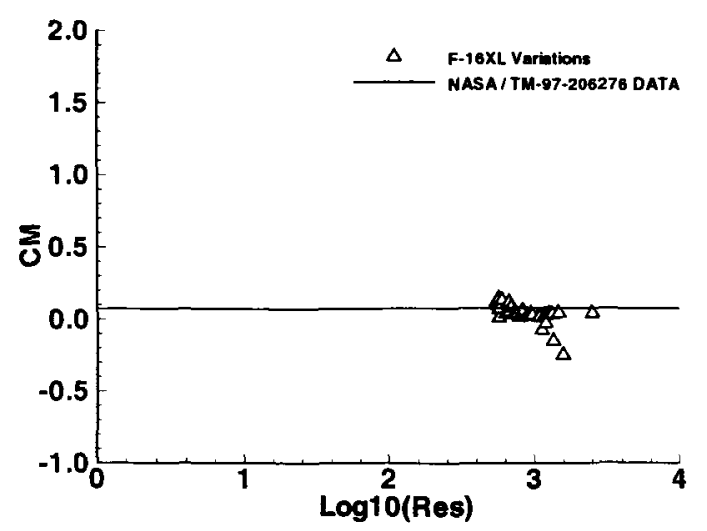

Figure 10. Variation in computed pitching moment coefficient with surface resolution for the $\mathrm{F}-16 \mathrm{XL}$ configurations.

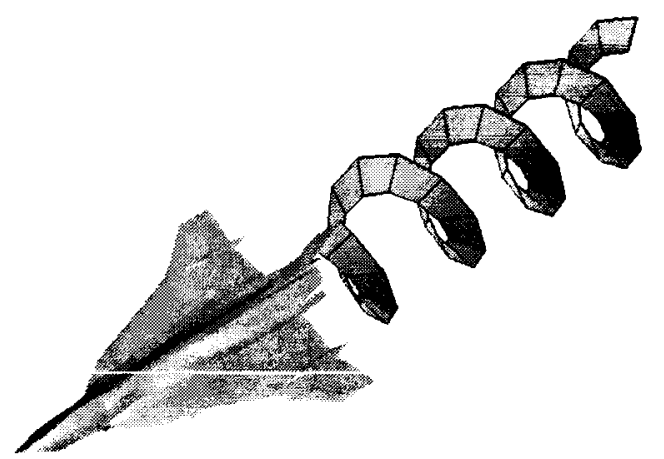

Figure 11. Sample body axis pure rotary roll maneuver, with trailing vertical tail wake.

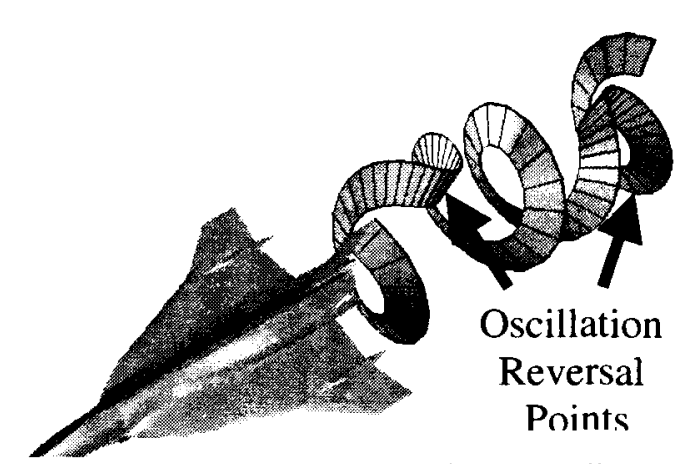

Figure 12. Sample body axis oscillatory roll maneuver, with trailing vertical tail wake. 


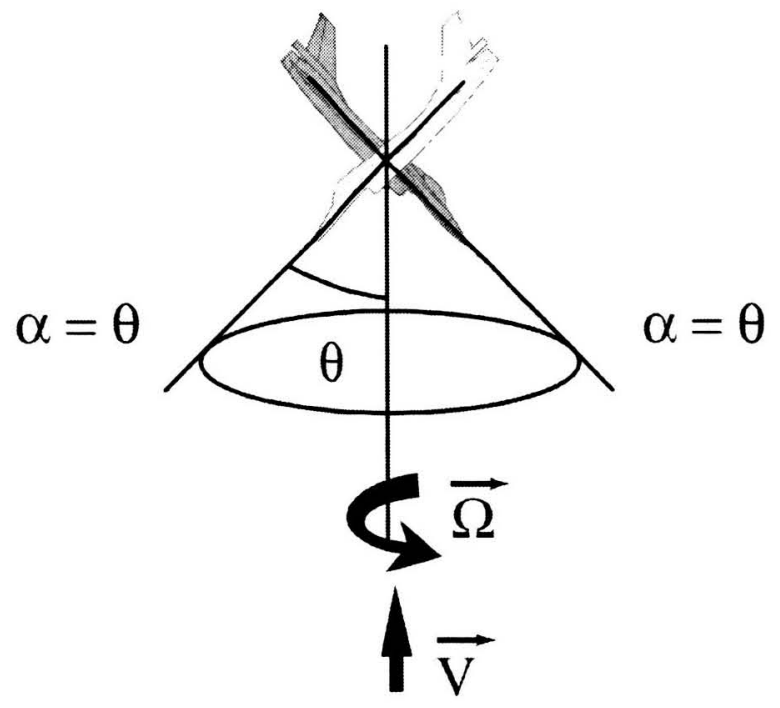

Figure 13. Conventional coning motion.

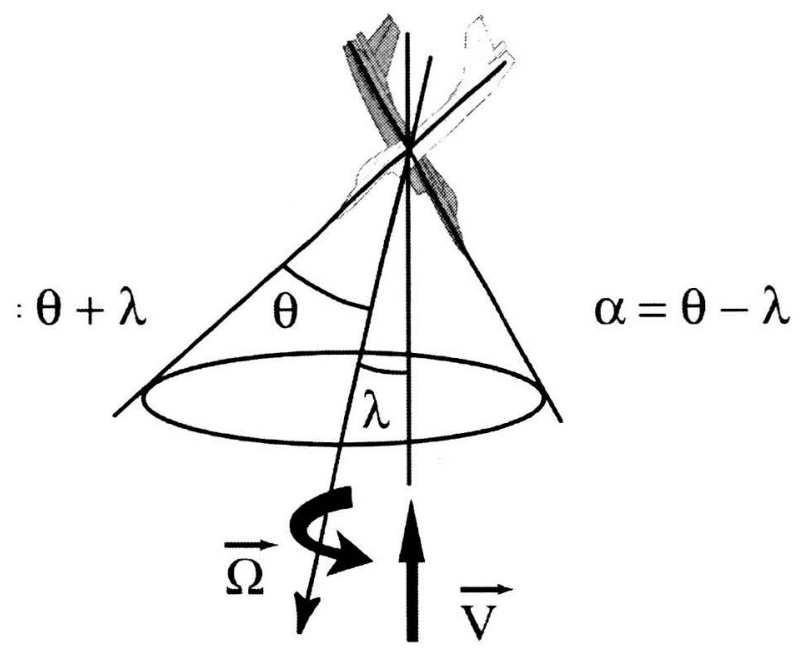

Figure 14. Oscillatory coning, or inclined axis roll (IAR), motion.

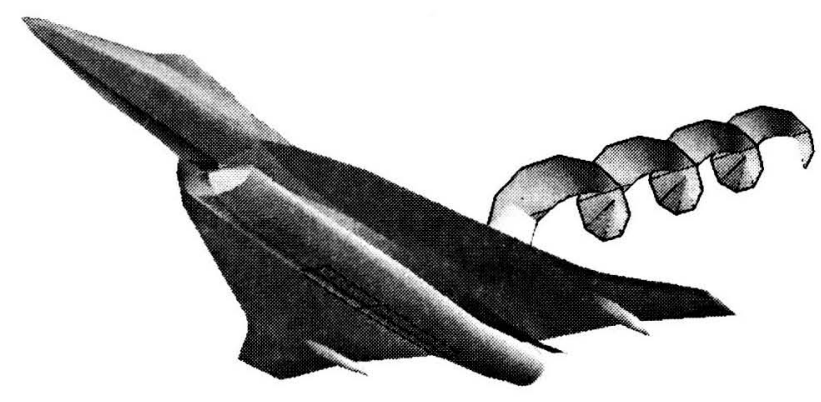

Figure 15. Computational illustration of pure coning motion.

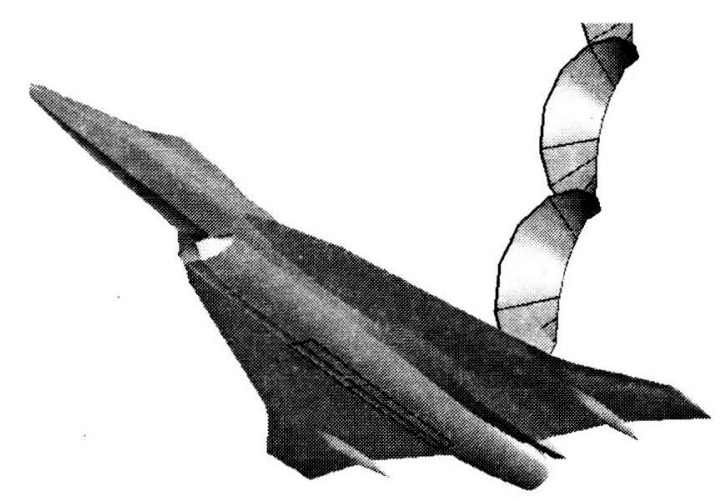

Figure 16. Computational illustration of oscillatory coning, or inclined axis roll (IAR), motion. 